\title{
Self-Compensating Liquid-Repellent Surfaces with Stratified
}

\section{Morphology}

Songtao Hu, ${ }^{\dagger}$ Xiaobao Cao, ${ }^{\ddagger}$ Tom Reddyhoff, ${ }^{\S}$ Debashis Puhan, ${ }^{\S}$ Sorin-Cristian Vladescu, ${ }^{\S}$ Qian Wang, ${ }^{\S}$ Xi Shi, ${ }^{,, \dagger}$ Zhike Peng, ${ }^{*,}$ Andrew J. deMello, ${ }^{\ddagger}$ and Daniele Dini ${ }^{\S}$

"State Key Laboratory of Mechanical System and Vibration, Shanghai Jiao Tong University, Shanghai 200240, China

${ }^{\star}$ Department of Chemistry and Applied Biosciences, ETH Zurich, Zurich 8093, Switzerland

${ }^{\S}$ Department of Mechanical Engineering, Imperial College London, London SW7 2AZ, United Kingdom

ABSTRACT: Artificial liquid repellent surfaces have recently attracted vast scientific attention; however, achieving mechanical robustness remains a formidable challenge before industrialization can be realized. To this end, inspired by plateaus in geological landscapes, a self-compensating strategy is developed to pave the way for the synthesis of durable repellent surfaces. This selfcompensating surface comprises tall hydrophobic structural elements, which can repel liquid droplets. When these elements are damaged, they expose shorter structural elements that also suspend the droplets and thus preserve interfacial repellency. An example of this plateau-inspired stratified surface was created by 3D direct laser lithography micro-nano fabrication. Even after being subjected to serious frictional damage, it maintained static repellency to water with a contact angle above $147^{\circ}$ and was simultaneously able to endure high pressures arising from droplet impacts. Extending the scope of nature-inspired functional surfaces from conventional biomimetics to geological landscapes, this works demonstrates that the plateau-inspired self-compensating strategy can provide an unprecedented level of robustness in terms of sustained liquid repellency.

KEYWORDS: artificial surface, 3D laser lithography, liquid repellency, mechanical robustness, friction

\section{INTRODUCTION}

Natural surfaces, serving as the interface between an organism and its surroundings, have to fulfill inherent functions responding to environmental physicochemical reactions by optimizing morphological structure and chemical composition. As a common pathway in biomimetics, understanding and mimicking morphological and chemical attributes from biological evolution provides an ideal strategy to translate fascinating functions of natural surfaces into synthetic systems. ${ }^{1}$ One interesting natural inspiration is the liquid repellent nature of surfaces, which has attracted great attention because of its scientific and economic importance in applications of selfcleaning, ${ }^{2}$ antifogging, ${ }^{3}$ anti-icing, ${ }^{4}$ antireflection, ${ }^{5}$ water harvesting, ${ }^{6}$ bio-analysis, ${ }^{7}$ microdroplet manipulation, ${ }^{8}$ etc. The most well-known example is the lotus effect that exhibits water repellency 
by combining hierarchical morphology and wax-based chemical decoration, ${ }^{9}$ with the former serving as the crucial factor. However, hierarchical surfaces, even if embellished by chemical modification of low-surface-energy materials, still encounter limitations that prevent mature industrialization. For instance, most repellency is effective for aqueous liquids but fails for lowsurface-tension liquids such as oils and organic solvents; moreover, repellency is usually restricted to static conditions relating to droplet condensation at high relative humidity and is limited in kinetic circumstances associating with high-velocity impacting events such as rain droplets. Excitingly, springtails, the most widespread arthropods on Earth, ${ }^{10}$ present a mushroom-like geometry on their cuticles, which has evolved to avoid wetting by water and organic liquids so as to guarantee a dermal respiration in aqueous habitats and temporarily flooded environments. This mushroom-like topology has contributed to the ideation of a competitive design employing singly/doubly/triply reentrant head structures atop supporting pillars, ${ }^{11-19}$ overcoming the aforementioned limitations even regardless of chemical modification.

Mechanical robustness is a major challenge in designing and processing artificial repellent surfaces and is regarded as limiting the uptake of pillar-based surfaces in commercial applications. ${ }^{10}$ Interfacial repellency usually diminishes after a period of time due to breakages occurring at the base and the nick under a shear load. In general, hard materials such as metals and ceramics seem to be a solution to improve structural durability; however, they can trigger an even worse resistance than soft materials owing to the loss of compliance. Thus, self-supporting networks have been added to interconnect adjacent underlying structures so as to enhance the mechanical stability in comparison to an array of individual supporting structures. ${ }^{15}$ In order to recover repellency which would otherwise be reduced by these additive self-supporting networks, the fabrication of an undulated substrate is strongly recommended. ${ }^{18}$ Another approach elaborated to improve interfacial durability is the self-healing concept, whereby preserving agents encapsulated in structural pores can quickly migrate into the damaged regions. ${ }^{20,21}$ For this self-healing strategy, more studies are further required by interfacial structures under a regular pattern in comparison to the existing scenario with the structures following a random distribution.

Here, we report a peculiar artificial surface inspired by bi-Gaussian stratified plateaus existing in natural landscapes and engineering industries, whose realization and response is shown to provide a promising morphological, self-compensation strategy to improve interfacial durability (see Figure 1). Unlike the aforementioned self-supporting and self-healing strategies, the short structural elements on our surface will automatically inherit the repelling roles played by the taller ones when the latter are damaged due to mechanical contact and friction, thereby ensuring stable and sustained liquid repellency. Using 3D direct laser lithography micro-nano fabrication, we have realized our plateau-inspired self-compensating design and have demonstrated its water repellency in both static and kinetic circumstances following mechanical damage induced by sliding friction. 


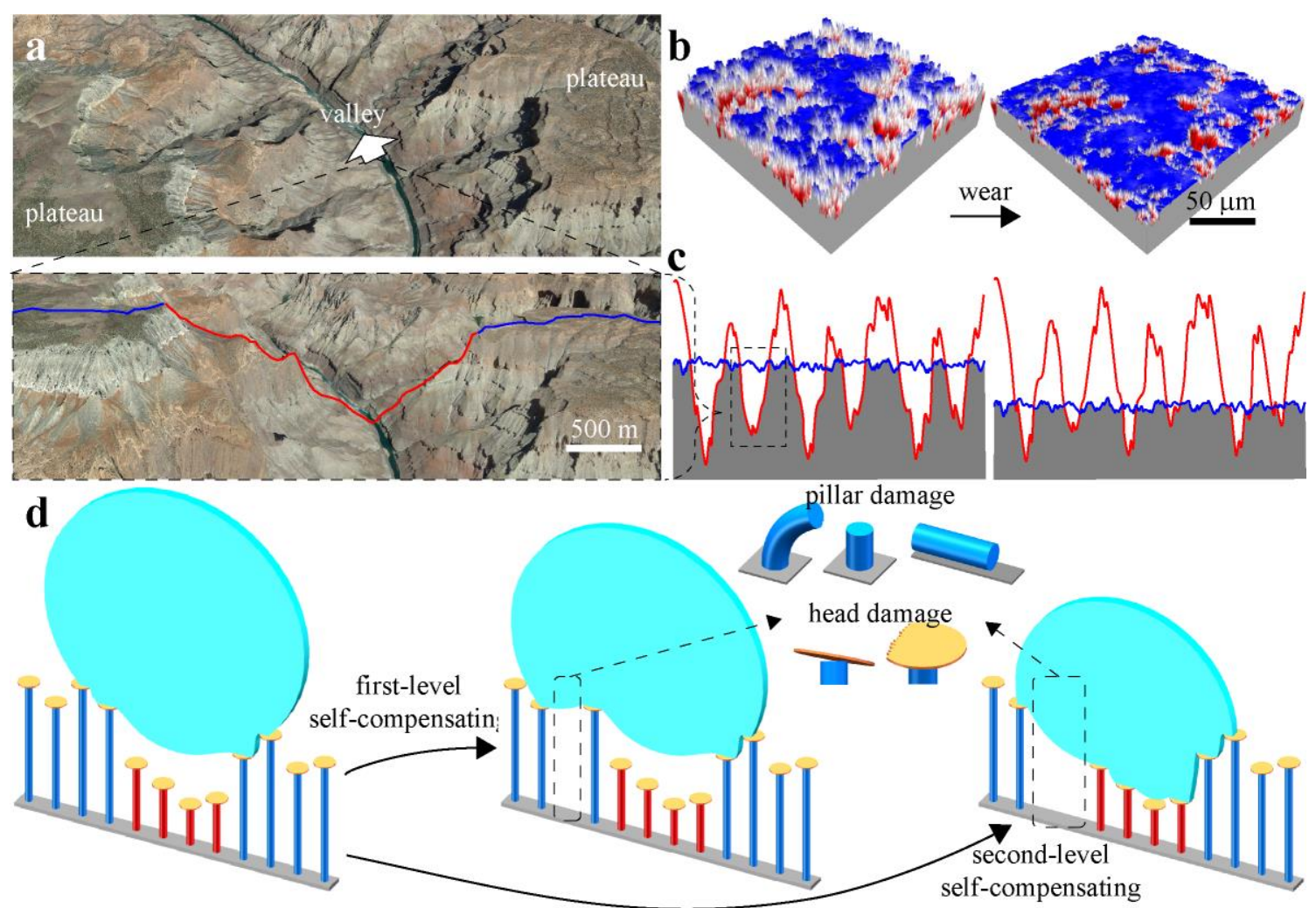

Figure 1. Ideation of self-compensating liquid repellent surface. (a) Bi-Gaussian stratified morphology of plateaus observed in the Grand Canyon National Park from top and side views. (b) Bi-Gaussian stratified morphology observed on engineering surfaces before/after frictional wear. (c) 2D schematic diagram of a bi-Gaussian stratified morphology to ensure a stable and sustained wear resistance. (d) Schematic diagram of a self-compensating surface with underlying pillars following a bi-Gaussian stratified height distribution to prolong the liquid repellency of mushroompillar structures.

\section{EXPERIMENTAL SECTION}

2.1 Surface Modelling. The simulation method of bi-Gaussian stratified morphology (see Section S1) was implemented in Matlab (Mathworks Inc, USA) to generate the pillar-height matrix according to the prescribed bi-Gaussian stratified indexes (see Table S1). Mushroom-pillar structures were modelled in SolidWorks (Dassault Systemes, France) whose heights were set as the above matrix. The resulting 3D model of the plateau-inspired surface was output at a stereolithography format. This approach was also adopted to model the other four plateau-inspired surfaces with emulated mechanical damage.

2.2 Surface Fabrication. On Photonic Professional System (NanoScribe GmbH, Germany), the IP-S photoresist (NanoScribe GmbH, Germany) was exposed to a 780-nm femtosecond laser through an oil-immersion objective at a speed of $100 \mathrm{~mm} \cdot \mathrm{s}^{-1}$ with a power of $110 \mathrm{~mW}$ so as to be fabricated on an ITO coated fused silica. The laser was commanded to write $0.5 \mu \mathrm{m}$ beneath the interface between the photoresist and the substrate, thus ensuring a strong adhesion between the printed structures and the glass substrate. The fabrication was then developed for 20 minutes in SU- 
8 Developer (MicroChem Corp, USA) and rinsed in isopropyl alcohol and deionized water.

2.3 Morphology Characterization. The morphologies of fabricated surfaces were inspected by MXB-2500REZ (Hirox Europe Ltd, France) and S-3400N SEM (Hitachi Ltd, Japan) accounting for a multi-scale requirement.

2.4 Contact Angle Measurement. The contact angle of water droplets ( $4 \mu \mathrm{L})$ was measured on Rame-Hart Contact Angle Goniometer (Rame-Hart Instrument Co, USA) in a sessile drop mode under controlled temperature $\left(20{ }^{\circ} \mathrm{C}\right)$ and relative humidity $(45 \%)$. Each contact angle was captured 30 seconds after the droplet contacted the fabricated surface so as to ensure equilibrium. Also, a water droplet ( $4 \mu \mathrm{L}$ ) was created and held by the syringe above the fabricated surface so as to measure the contact angle hysteresis through capturing the advancing and receding angles by sliding the positioning platform at a velocity of $100 \mu \mathrm{m} \cdot \mathrm{s}^{-1} .{ }^{14}$ The measurement was conducted five times on the same fabricated surface for a repeatability.

2.5 Droplet Impacting Test. The spreading, retracting and after-retracting behaviors after water droplets impacted the fabricated surface were captured by Phantom Miro ex2 (AMETEK Inc, USA) assembled with Zoom 7000 (Navitar Inc, USA) with a sampling rate of $2900 \mathrm{fps}$. A micro syringe pump was used to release water droplets with a volume of $4 \mu \mathrm{L}$. The height to release the droplets was changed to realize the adjustment of $W e$.

2.6 Friction Test. The friction test was conducted by loading and sliding a triangular rubber wiper against the printed surface on CETR UMT (Bruker Corp, USA). This equipment was operated in a pin-on-disc mode, so that the printed surface was held stationary as the lower specimen, while the wiper had a controlled movement as the upper specimen. The wiper was declined to generate a line contact with the printed surface near one edge at a normal load of $0.2 \mathrm{~N}$, and then was slid over the surface at a speed of $1 \mathrm{~mm} \cdot \mathrm{s}^{-1}$ at an unchanged normal distance. The normal and frictional loads were recorded at a step of $5 \mathrm{~ms}$. After the friction test, the printed surface was rinsed in isopropyl alcohol and deionized water.

\section{RESULTS AND DISCUSSION}

3.1 Ideation of Self-Compensating Design. To achieve stable and sustained repellency, we designed an artificial self-compensating surface with its structural elements composed of mushroom heads atop pillars following a bi-Gaussian stratified height distribution, inspired by plateau morphologies existing in geological landscapes (see Figure 1a) and engineering industries (see Figure 1b). Similar to the fractal concept motivated by natural landscapes (e.g. coastlines), a bi-Gaussian stratified morphology can be clearly observed on plateaus such as in the Grand Canyon National Park, where the initial mountains generated in past diastrophisms have been truncated by environmental actions of wind and rain so as to engender flat plateaus interpenetrated by deep valleys. In fact, this peculiar morphology can be also found on engineering surfaces subjected to frictional wear, ${ }^{22,23}$ and has been modelled to a large-scale roughness (see red lower component in Figure 1c) intersected by a small-scale roughness (see blue upper component) with each component individually respecting a Gaussian height distribution due to the randomness of a wear process. Notably, as opposed to the classical fractal characteristic emphasizing the self-similarity at different scales, the bi-Gaussian one renders its stratified property (i.e., retaining the lower height in the 
combination of the upper and lower components) at a single scale.

Researchers have long overlooked the real-life bi-Gaussian stratified nature of surfaces because of its simplification to a single-stratum, non-Gaussian modelling (e.g., exponential). However, the bi-Gaussian stratified characteristic, recently, has captivated scientists and engineers for its impressive functions arising from its particular stratified topology in comparison to a uniform height or a Gaussian distribution in terms of friction ${ }^{22,23}$ and wettability. ${ }^{24,25}$ Looking at the frictional field, as the upper component plays critical roles in load bearing and wear resistance while the lower component serves as lubricant reservoirs and debris traps, the performance of an engineering surface can be manually designed by controlling the configuration of the two components. ${ }^{22}$ Specifically, the upper component of an unused engineering surface is designed to individually resist the opponent body at the start of the service; then, along with the frictional wear, the mean height of the upper component declines gradually relative to the lower component, making more and more surface nodes transform into the upper component (see Figures $1 \mathrm{~b}$ and $1 \mathrm{c}$ ) so as to gain a stable and sustained wear resistance. ${ }^{23}$ Accordingly, this noteworthy attribute of a bi-Gaussian stratified morphology triggers a window of opportunity for a self-compensating strategy to improve the durability of springtail-inspired repellent surfaces (see Figure 1d). More precisely, all mushroom-pillar elements on an artificial surface can be divided into two layers in terms of element heights, where the upper-layer elements are well designed to guarantee liquid repellency; with the repellent loss of a upper-layer element induced by mechanical damage under a slight shear load, other upper-layer elements will be exposed to grab the role of the broken one (termed as the firstlevel self-compensating); when most upper-layer elements lose their repellency under a heavy shear load, some elements on the lower layer will be activated (termed as the second-level selfcompensating). As opposed to mechanically robust surfaces by use of a hierarchical design based on a multiscale, fractal characteristic, ${ }^{26}$ the single-scale, stratified property of our bi-Gaussian characteristic provides a purely microscale solution to improve the mechanical robustness of liquid repellent surfaces.

3.2 Design and Fabrication of Self-Compensating Surface. A self-compensating surface (see Figure 2a) was purposely designed as a $5 \times 5 \mathrm{~mm}^{2}$ matrix consisting of $10 \times 10$ units, each unit encompassing $8 \times 8$ elements with mushroom heads atop pillars (namely, element distance $p$ equals to $62.5 \mu \mathrm{m}$ ). The reason for such a matrix design was related to the scientific demand on eliminating the repetitive error caused by different contacting positions in the following static and kinetic repellent experiments. For each element, a doubly reentrant head was utilized by setting $d_{0}$ to 35 $\mu \mathrm{m}, h_{1}$ to $2 \mu \mathrm{m}, h_{2}$ to $4 \mu \mathrm{m}$ and $w$ to $1 \mu \mathrm{m}$. Meanwhile, the heights of underlying pillars $\left(d_{1}=10\right.$ $\mu \mathrm{m})$ in each unit were designed to follow a bi-Gaussian stratified distribution, thus yielding an obvious two-layer arrangement. The pillar heights were prescribed by bi-Gaussian stratified indexes (defined in Figure S1) including the root-mean-square height of upper-layer pillars $S p q$, the root-mean-square height of lower-layer pillars $S v q$, and the proportion of upper-layer pillars $S m q$. In addition, an extra reinforced structure was introduced to strengthen the mechanical property at the pillar bottom as well as to intensify the adhesion between the pillar bottom and the substrate. Also, a fillet was employed accounting for a smooth transition between the reinforcement and the 
pillar to eliminate the shear load arising from an abrupt shape change.

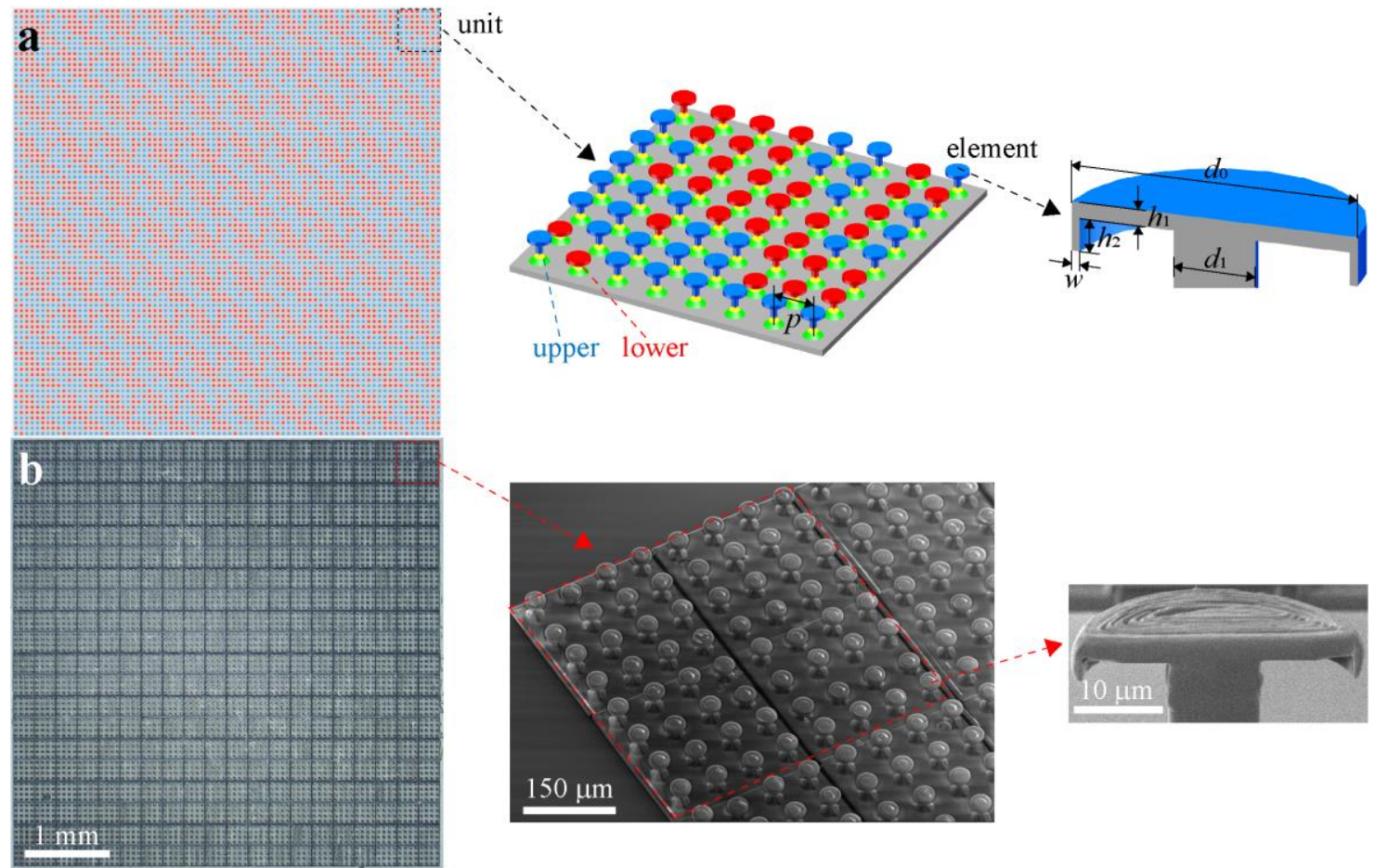

Figure 2. Self-compensating liquid repellent surface. (a) Design of a self-compensating surface as a matrix consisting of $10 \times 10$ units, each unit encompassing $8 \times 8$ mushroom-pillar elements following a bi-Gaussian stratified height distribution. (b) Fabrication of the self-compensating surface.

The designed surface was realized with 3D direct laser lithography on Photonic Professional System (NanoScribe GmbH, Germany) by fabricating the target structures made of IP-S photoresist (NanoScribe GmbH, Germany) on an ITO coated fused silica via a two-photon polymerization (see Figure 2b). Notably, the NanoScribe system can be regarded as the most precise rapid prototyping technology to tailor 3D structures at a micrometric level even at a nanoscale. This up-to-date technology has been successfully applied in optics, ${ }^{27}$ photonics ${ }^{28}$ microfluidic, ${ }^{29}$ biology, ${ }^{30}$ mechanical metamaterials, ${ }^{31}$ etc., and has recently paved the way to shed light on wetting mechanism. ${ }^{6,19,25,32-34}$ Thanks to the optimization of structural design and processing technic (see Figure S2), artificial mushroom-pillar structures were strongly attached to glass substrates in a great consistency with the initial design, thereby indicating our fabrication approach to be an effective avenue to combine the springtail- and plateau-inspired topologies on a single functional surface.

3.3 Water Repellency with Emulated Mechanical Damage. To reveal the self-compensating mechanism of the above artificial surface, in addition to its intact state, we also designed and fabricated another four states to emulate an evolution where mechanical damage was assumed to propagate from high to low due to contact and friction (see Figure 3). Among these four states, the 
top $12.5 \%$ and $25 \%$ of mushroom heads first faded away; second, the top $50 \%$ of mushroom heads vanished, equaling to a full mushroom-head disappearance on the upper layer; third, all the underlying pillars were exposed, indicating a thorough failure of mushroom heads over the whole surface. In comparison to real mechanical damage under a frictional wear, such an emulating approach provides an alternative to exactly control the degree of mechanical deterioration so as to establish the relation between the damage degree and liquid repellency, thereby quantitatively assessing the self-compensating capacity.

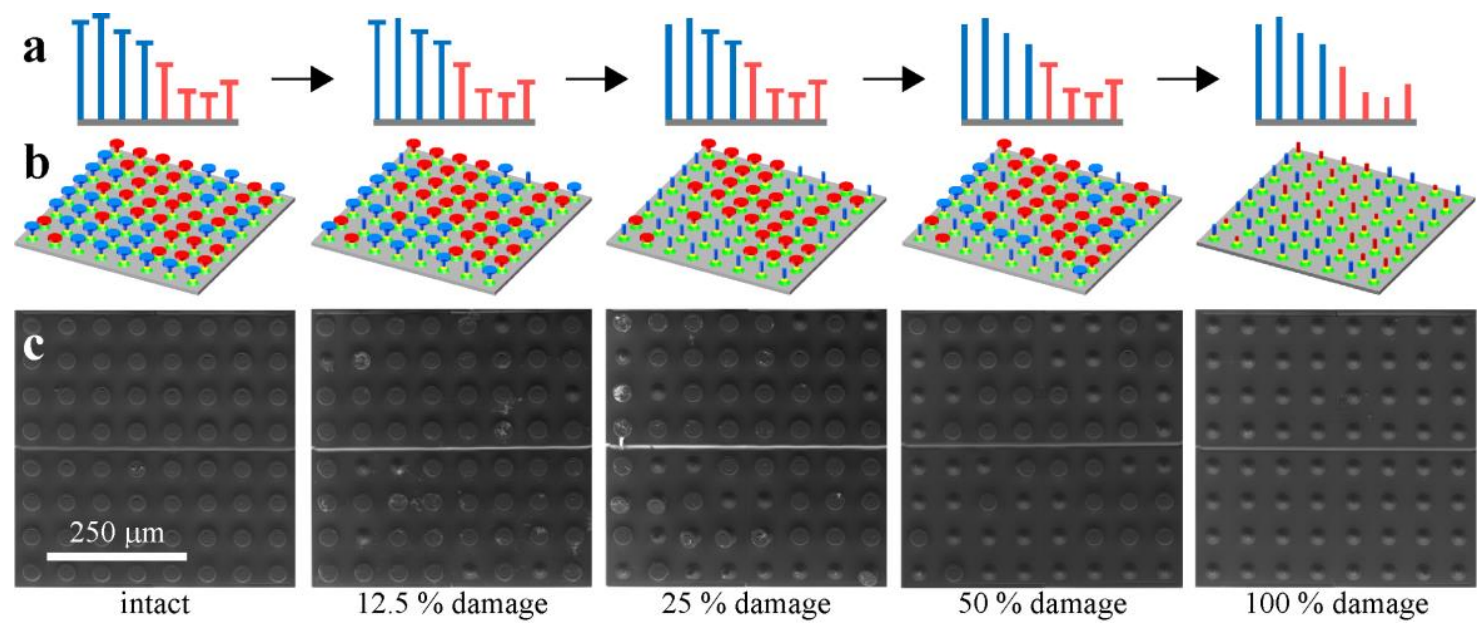

Figure 3. Self-compensating liquid repellent surface in an emulated evolution of mechanical damage. (a) Schematic diagram of an emulated evolution of mechanical damage due to contact and friction, where a mushroom-head disappearance propagated from high to low: intact, $12.5 \%$ damage, $25 \%$ damage, $50 \%$ damage, and complete damage. (b,c) Unit design and fabrication of the self-compensating surface with emulated mechanical damage, where fabrication images were captured after static and kinetic repellent experiments, indicating an excellent structural strength.

3.3.1 Static Water Repellency. The intact surface exhibited a perfect anti-penetrating ability to water droplets $\left(156.4^{\circ}\right.$ in Figure $\left.4 a\right)$ even realized with a hydrophilic photoresist (contact angle on a smooth plane made of the same photoresist was $67.2^{\circ}$ ), thus verifying the impressive capacity of a mushroom-pillar combination to transform the initial hydrophilicity into a macroscopic superhydrophobicity. Referring to the well-known Wenzel theory, a roughness will amplify the inherent hydrophilicity as the result of an area extension. The converse effect here was ascribed to not only the mushroom heads ${ }^{11-19}$ but also the Fakir effect ${ }^{35-37}$ provided by the pillars with a large aspect ratio. Namely, the droplet no longer conformed to the substrate but rested on top of the pillars while gas pockets were simultaneously trapped underneath the droplet. Gratifyingly, along with the emulated mechanical damage, the surface clearly presented a great self-compensating ability. More precisely, the contact angle retained at $156.5^{\circ}$ with $12.5 \%$ damage, reduced to $95.0^{\circ}$ with $25 \%$ damage, and arrived at $93.2^{\circ}$ to approach the hydrophobic-hydrophilic transition with $50 \%$ damage. Of note, even when all upper-layer mushroom heads disappeared (50\% damage), the repellence still held a hydrophobicity owing to the existence of an intact lower layer that individually 
undertook the repellent task. The repellency finally reached a hydrophilic value $\left(87.6^{\circ}\right)$ in the circumstance of a complete loss on mushroom heads, thus verifying the Fakir effect of pillars in comparison to the value of $67.2^{\circ}$ on a smooth plane. Moreover, the contact angle hysteresis was captured at an unchanged value of $53.1^{\circ}$ when the damage percentage increased from $0 \%$ to $12.5 \%$. When the damage percentage continued to raise, the droplet got rid of the constraints from the syringe but kept relative rest with the sliding platform, thereby being unable to capture the advancing and receding angles.
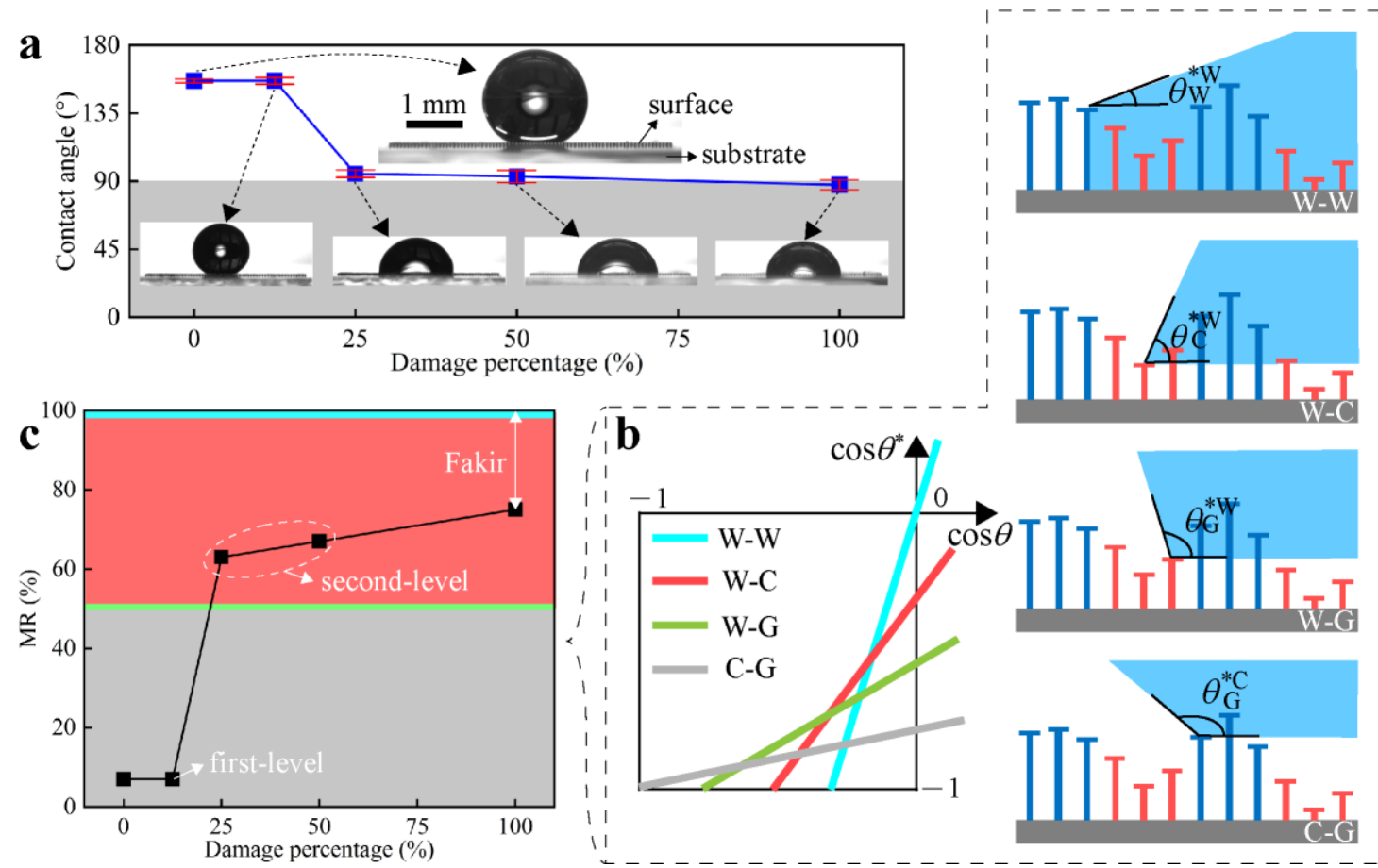

Figure 4. Static water repellency of the self-compensating surface with emulated mechanical damage. (a) Contact angles of water droplets. (b,c) Wetting states of the upper and lower layers by using a bi-Gaussian stratified wetting model consisting of four wetting sub-states, i.e., W-W, W-C, W-G and C-G.

For a further assessment of the self-compensating capacity given by a bi-Gaussian stratified arrangement, we established a theoretical model based on the most extant Wenzel and Cassie theories to link the intrinsic contact angle $\theta$ and apparent contact angle $\theta^{*}$ (see Figure $4 \mathrm{~b}$ which was clearly modelled in Section S3). As opposed to previous studies, in this model, material ratio (MR), indicating the probability to find an interfacial element with its height above a specified value, was introduced to define the boundary between the wetted and unwetted regions. In other words, the elements with their pillars higher than a specified height are wetted, while otherwise are arid (see Figures S3 and S4). Consequently, the macroscopic Wenzel and Cassie states of liquid droplets on a plateau morphology has been divided into four sub-states. In the W-W state, the value of MR is $100 \%$ with the lower and upper layers both in the Wenzel state; in the W-C state, the MR equals to a value located in the interval of (Smq, $100 \%)$ with the upper layer in the Wenzel state and the 
lower layer in the Cassie state; in the W-G state, the MR right equals to Smq, indicating the upper layer in the Wenzel state but the lower layer in a gas environment; in the C-G state, the MR reaches a tiny value in the interval of $(0, S m q)$ in which the lower layer stays in the gas environment while the upper layer has entered into the Cassie state. Of course, the value of MR is strongly dependent on the physicochemical attributes of solid and liquid as well as environmental factors, making it a challenge to be theoretically calculated. ${ }^{25}$ Anyhow, unlike the previous Wenzel and Cassie models, our model can be used as a crucial probe to estimate the wetting states of the two component layers, as similar to some multiscale wetting models established to distinguish the different wetting states of micro and nanostructures for hierarchical surfaces. ${ }^{6,8,38}$ The measured apparent contact angles in Figure $4 \mathrm{a}$ and intrinsic contact angle $\left(67.2^{\circ}\right.$ on a smooth plane) were plotted to estimate the values of MR for the self-compensating surface with different mechanical damage (see Figure S5), and finally summarized in Figure 4c. In the intact or $12.5 \%$ damage circumstance, only $7 \%$ of structural elements was wetted, indicating the C-G state (note that the $S m q$ value of the selfcompensating surface was designed to $50 \%$ as listed in Table S1); the ratio of wetted elements raised up to $63 \%$ and $67 \%$ when the damage percentage raised up to $25 \%$ and $50 \%$, indicating the W-C state; with a complete loss of mushroom heads, the surface still located in the W-C state rather than the W-W state because of the Fakir effect of pillars. The unchanged MR when the damage percentage ranged from $0 \%$ to $12.5 \%$ indicated a great first-level self-compensating ability supported by the upper layer, meanwhile the MR translated to $63 \%$ and $67 \%$ when the damage percentage increased to $25 \%$ and $50 \%$, thus disclosing the second-level self-compensating capacity ascribed to the uninjured lower layer.

3.3.2 Kinetic Water Repellency. In addition to the static circumstance, the self-compensating capacity of the designed surface should be inspected for kinetic repellency to impinging droplets. ${ }^{4,7,14,18}$ We therefore conducted an impacting test to investigate the spreading, retracting and after-retracting behaviors of water droplets with different impacting velocities (see Movie S1). Figure 5a presented the maximum spreading factor $D_{\max } / D_{0}$ as a function of Weber number $(\mathrm{We})$ on the self-compensating surface with emulated mechanical damage. Herein, We, defined as $D_{0} \rho V_{0}^{2} / \sigma$, is a dimensionless pressure index to quantify the ratio between the inertial and capillary forces, where $D_{0}$ and $V_{0}$ are the diameter and impacting velocity of a droplet in flight before the contact instant, and $\rho$ and $\sigma$ are the density and surface tension of the droplet. $D_{\max }$ is the maximum droplet diameter before the retracting behavior. Up to now, the relationship between $D_{\max } / D_{\max }$ and $W e$ is still a heated subject of controversy because of an open debate on two different scaling laws relying on energy conservation $\left(D_{\max } / D_{0} \propto W e^{0.5}\right)$ and momentum-mass conservation $\left(D_{\max } / D_{0} \propto\right.$ $\left.W e^{0.25}\right) .{ }^{39-42}$ In our study, the latter momentum-mass conservation was preferred (see Figure S6). The intact surface exhibited a greater maximum spreading factor than other damaged cases at the same $W e$, indicating a weakened drag in the spreading phase owing to a mushroom-like topology. 

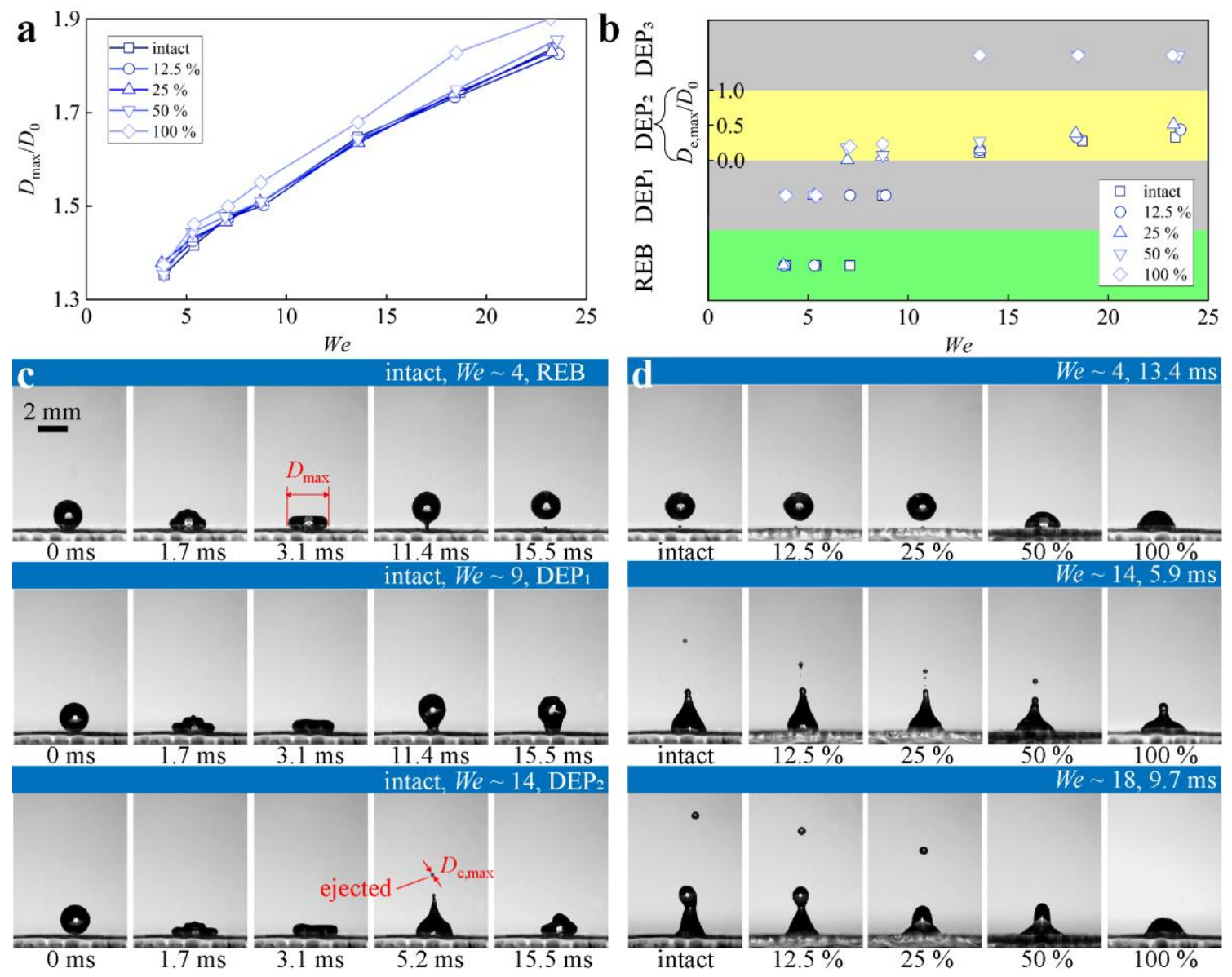

Figure 5. Kinetic water repellency of the self-compensating surface with emulated mechanical damage. (a) Maximum spreading factor $D_{\max } / D_{0}$ as a function of $W e$. (b) After-retracting behaviors including rebounding (REB) and depositing $\left(\mathrm{DEP}_{1}, \mathrm{DEP}_{2}\right.$ and $\left.\mathrm{DEP}_{3}\right)$ as a function of We. (c) Snapshots to illustrate different after-retracting behaviors. d) Snapshots to show the impact of damage degree on after-retracting behaviors.

We further investigated the after-retracting behaviors of water droplets and gained a considerable regularity translating from rebounding (REB) to depositing (also from $\mathrm{DEP}_{1}, \mathrm{DEP}_{2}$ to $\mathrm{DEP}_{3}$ ) with an increased $W e$, as summarized in Figure $5 \mathrm{~b}$. In the intact state, a rebounding was observed at $W e \sim 4$ (visualized in Figure $5 \mathrm{c}$ ), thus indicating a water resistance to the pressure generated from an impacting event. However, this rebounding motion disappeared when $W e$ raised up to $\sim 9$, rendering a depositing response to an impact. When the value of We continued to raise over $\sim 14$, a depositing with the base part pinning onto the surface but the top part ejecting as satellites was observed. We further quantified it by characterizing the maximum diameter of the ejected satellites $D_{\mathrm{e}, \max }$ and successfully revealed an obvious monotonical increase along with an increased $W e$ value.

Figure $5 \mathrm{~b}$ also showed a considerable regularity of after-retracting behaviors translating from $\mathrm{REB}, \mathrm{DEP}_{1}, \mathrm{DEP}_{2}$ to $\mathrm{DEP}_{3}$ along with an intensified mechanical damage. At $W e \sim 4$ (visualized in Figure $5 \mathrm{~d}$ ), the surface subjected to $12.5 \%$ or $25 \%$ damage still kept a rebounding response to the 
dripping droplets, highlighting a great self-compensating ability. However, the after-retracting behavior has switched to a $\mathrm{DEP}_{1}$ state when the damage percentage arrived at $50 \%$ or $100 \%$. When We increased to $\sim 7$, the surface started to exhibit a $\mathrm{DEP}_{2}$ behavior with the damage ratio higher than $25 \%$. When We arrived at $\sim 14$, the surface presented a $\mathrm{DEP}_{2}$ action in the intact, $12.5 \%, 25 \%$ and $50 \%$ damage circumstances, where the size of the ejected satellites was proportional to the damage degree. In addition, the dominated role played by the damage degree can be also demonstrated by the $\mathrm{DEP}_{3}$ phenomenon with $100 \%$ damage. In comparison to the fully damaged case, the $\mathrm{DEP}_{3}$ behavior started to appear with $50 \%$ damage when $W e$ raised up to $\sim 18$, indicating that a relatively severer damage degree was easier to receive a $\mathrm{DEP}_{3}$ response. In sum, it can be concluded that a mechanical damage of mushroom heads will weaken the kinetic repellency, thus translating the after-retracting behaviors from $\mathrm{REB}, \mathrm{DEP}_{1}, \mathrm{DEP}_{2}$ to $\mathrm{DEP}_{3}$. However, our surface can delay this damage-induced translation based on its self-compensating capacity.

3.4 Water Repellency with Real Mechanical Damage. We applied the self-compensating mechanism drawn from the above emulated investigation to real mechanical damage caused by frictional wear (see Figure 6a). A triangular rubber wiper was declined to approach a line contact with the underlying printed surface at a specified normal load $F_{\mathrm{z}}$ of $0.2 \mathrm{~N}$, and then was slid over the surface at a speed of $1 \mathrm{~mm} \cdot \mathrm{s}^{-1}$. In order to resolve the impact of a bi-Gaussian stratified arrangement of element heights on our self-compensating surface, we added a uniform reference which fully copied the stratified case except for uniform element heights. At the sliding stage of the friction test, the interaction of the mated pair (i.e., wiper and printed surface) under the normal load $F_{\mathrm{z}}$ produced a frictional load $F_{\mathrm{x}}$ around $0.2 \mathrm{~N}$, thereby making the coefficient of friction $F_{\mathrm{z}} / F_{\mathrm{x}}$ around 1 , which indicated a starving lubrication (usually implying a serious wear). The sliding stage led bending and missing postures to mushroom-pillar elements on the uniform surface, where the missing posture can be classified into mushroom loss and mushroom-pillar coupled loss. However, the stratified surface provided a stark contrast to the uniform one, where low-height elements maintained intact due to a stratified arrangement. Of note, a surface under a stratified pattern suggests fewer elements to support the opposite wiper in comparison to a uniform case, highlighting a stricter design rule (e.g., higher element density) required by our self-compensating surface. 


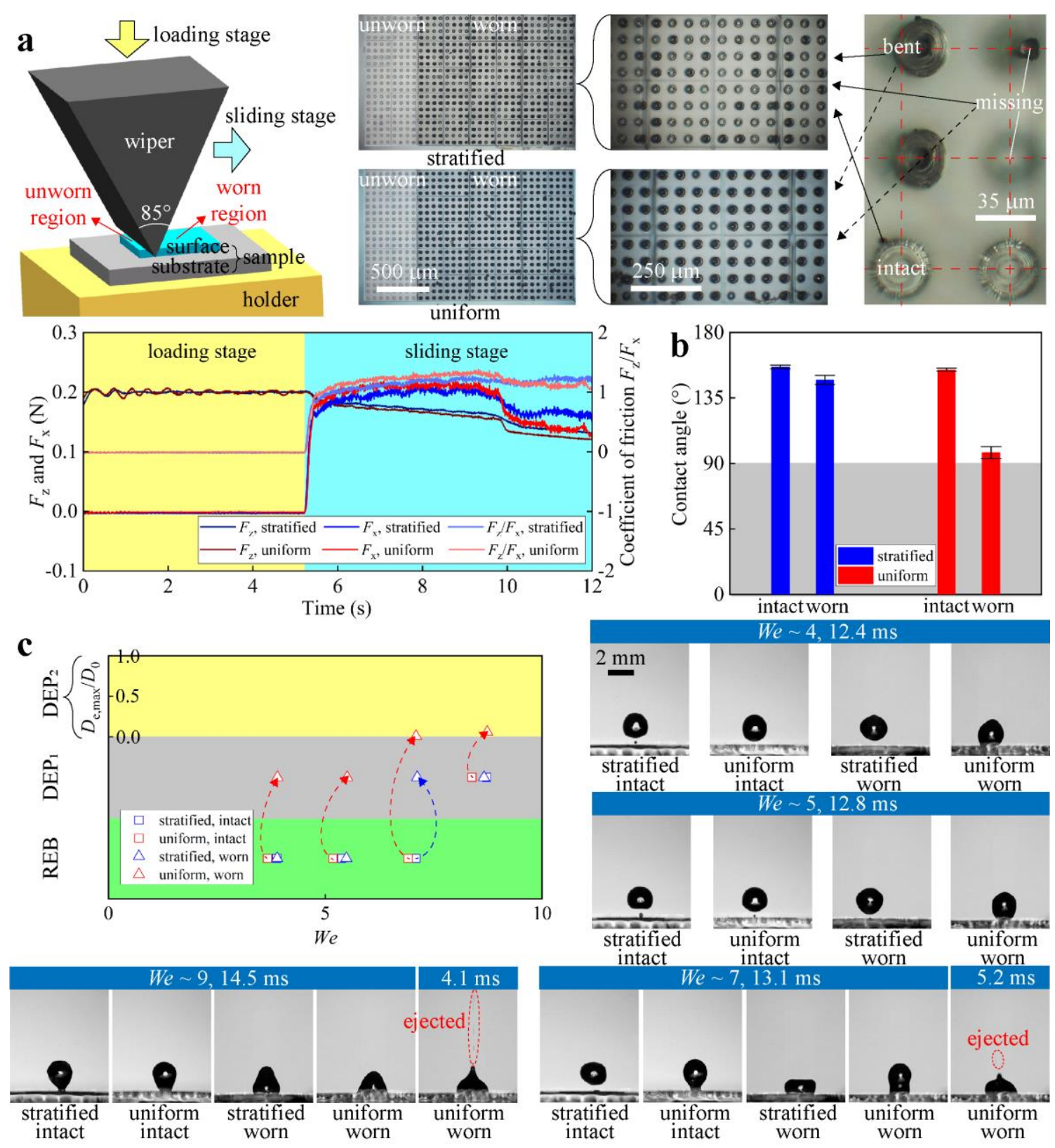

Figure 6. Water repellency of the self-compensating surface with friction-induced mechanical damage. (a) Sliding friction between a rubber wiper and printed surfaces, including the stratified surface and its uniform reference. (b) Contact angles of water droplets. (c) After-retracting behaviors of water droplets as a function of $W e$, and corresponding snapshots.

Gratifyingly, as depicted in Figure 6b, the contact angle of water droplets on the stratified surface subjected to frictional mechanical damage still kept at $147.4^{\circ}$, as opposed to the uniform case $\left(97.5^{\circ}\right)$ that has dramatically lost its superhydrophobicity to approach a hydrophilic state, thereby validating the self-compensating ability of a bi-Gaussian stratified arrangement in static circumstances. Looking at kinetic circumstances (see Figure 6c extracted from Movie S2), the stratified surface also provided a stark contrast to the uniform one in terms of after-retracting 
behaviors, indicating that the bi-Gaussian stratified arrangement can offer an unprecedented level of retaining the kinetic repellency regardless of frictional mechanical damage. More precisely, the worn stratified surface kept the original rebounding response at $W e \sim 4$ and $\sim 5$, while the uniform surface switched from REB to $\mathrm{DEP}_{1}$ due to the frictional mechanical damage; as the value of $W e$ raised up to $\sim 7$, the original $\mathrm{REB}$ behavior transformed into a $\mathrm{DEP}_{1}$ one on the worn stratified surface but into a $\mathrm{DEP}_{2}$ one on the worn uniform surface; when We reached $\sim 9$, the worn stratified surface retained the original $\mathrm{DEP}_{1}$ response, while the uniform surface transformed into a $\mathrm{DEP}_{2}$ condition from the original $\mathrm{DEP}_{1}$ state.

\section{CONCLUSIONS}

In summary, we were inspired by the bi-Gaussian stratified plateaus existing in natural landscapes and engineering industries to design artificial surfaces, which shows much promise in resolving the long-standing issue of improving the interfacial durability of liquid repelling surfaces using a selfcompensating strategy. In such a design, we used the springtail-inspired mushroom topology to decorate structural configuration to obtain liquid repellency while learned from the plateau-inspired stratified morphology to arrange structural elevation aimed at gaining a self-compensating capacity. 3D direct laser lithography was employed in the micro-nano fabrication to precisely replicate the mushroom configuration following a bi-Gaussian stratified height distribution. Unlike the previous self-supporting and self-healing strategies, on our self-compensating surface, short structural elements will automatically inherit the repelling roles played by taller ones when the latter are damaged by mechanical contact and friction, thereby ensuring stable and sustained liquid repellency. We investigated the self-compensating mechanism of our surface in both static and kinetic circumstances along with emulated mechanical damage propagating from high to low, finding that the surface, even with a relatively high structural damage (corresponding to $12.5 \%$ of the initial structures), exhibited a super static repellence with the contact angle above $150^{\circ}$ and was able to endure a high pressure arising from impinging droplets. Furthermore, we believe our work to be the first demonstration of a self-compensating strategy under friction-induced mechanical damage, providing an unprecedented level of control over the repellency for a stable and sustained service life. Our study also extended the scope of nature-inspired functional surfaces from conventional biomimetics to geological landscapes.

As an outlook, a detailed parametric discussion is further required to resolve the impact of different bi-Gaussian stratified indexes on optimizing the self-compensating capacity. As the element distance and autocorrelation length have been shown to play crucial roles in liquid repellency, optimizing the spatial characteristics of structural elements can be a potential avenue to drastically elevate the repellence regarding kinetic circumstances with extremely highly impacting events; this would contribute to extend the scope and the use of our self-compensating surfaces. Also, a surface under a stratified pattern usually suggests fewer elements to support the opposite body in comparison to a uniform case, demanding a stricter design rule for the spatial characteristics (e.g., high element density). In addition to the self-compensating inspiration in this study, the particular bi-Gaussian stratified arrangement also offers a flexible choice to tailor interfacial functions when the two layers are designed with different topologies or fabricated with dissimilar 
materials.

\section{ASSOCIATED CONTENT}

The Supporting Information is available free of charge on the ACS Publications website at DOI: xxxxxxxxxx.

Some more details about surface theories, surface design and fabrication, static liquid repellency, kinetic liquid repellency, and related figures (PDF)

Impacting test with emulated damage (Movie S1) (AVI)

Impacting test with friction-induced damage (Movie S2) (AVI)

\section{AUTHOR INFORMATION}

\section{Corresponding Authors}

*E-mail: xishi@sjtu.edu.cn (X.S.).

*E-mail: z.peng@sjtu.edu.cn (Z.P.).

\section{Notes}

The authors declare no competing financial interest.

\section{ACKNOWLEDGEMENTS}

This work was supported by China Postdoctoral Science Foundation (2019T120340, 2017M621458), National Natural Science Foundation of China (11572192, 11632011), and Engineering and Physical Sciences Research Council (EP/N025954/1).

\section{REFERENCES}

(1) Yao, L.; He, J. Recent Progress in Antireflection and Self-Cleaning Technology - from Surface Engineering to Functional Surfaces. Prog. Mater. Sci. 2014, 61, 93-143.

(2) Zhan, W.; Yu, S.; Gao, L.; Wang, F.; Fu, X.; Sui, G.; Yang, X. Bioinspired Assembly of Carbon Nanotube into Graphene Aerogel With "Cabbagelike" Hierarchical Porous Structure for Highly Efficient Organic Pollutants Cleanup. ACS Appl. Mater. Inter. 2018, 10, 1093-1103.

(3) Han, Z.; Feng, X.; Guo, Z.; Niu, S.; Ren, L. Flourishing Bioinspired Antifogging Materials with Superwettability: Progresses and Challenges. Adv. Mater. 2018, 30, 1704652.

(4) Bird, J. C.; Dhiman, R.; Kwon, H.; Varanasi, K. K. Reducing the Contact Time of a Bouncing Drop. Nature 2013, 503, 385-388.

(5) Raut, H. K.; Dinachali, S. S.; Loke, Y. C.; Ganesan, R.; Ansah-Antwi, K. K.; Gora, A.; Khoo, E. H.; Ganesh, V. A.; Saifullah, M. S. M.; Ramakrishna, S. Multiscale Ommatidial Arrays with Broadband and Omnidirectional Antireflection and Antifogging Properties by Sacrificial Layer Mediated Nanoimprinting. ACS Nano 2015, 9, 1305-1314.

(6) Tricinci, O.; Terencio, T.; Mazzolai, B.; Pugno, N. M.; Greco, F.; Mattoli, V. 3D Micropatterned Surface Inspired by Salvinia Molesta via Direct Laser Lithography. ACS Appl. Mater. Inter. 2015, 7, 25560-25567.

(7) Sun, Q.; Wang, D.; Li, Y.; Zhang, J.; Ye, S.; Cui, J.; Chen, L.; Wang, Z.; Butt, H.; Vollmer, D.; Deng, X. Surface Charge Printing for Programmed Droplet Transport. Nat. Mater. 2019, 18, 
936-941.

(8) Long, J.; Fan, P.; Gong, D.; Jiang, D.; Zhang, H.; Li, L.; Zhong, M. Superhydrophobic Surfaces Fabricated by Femtosecond Laser with Tunable Water Adhesion: from Lotus Leaf to Rose Petal. ACS Appl. Mater. Inter. 2015, 7, 9858-9865.

(9) Koch, K.; Bhushan, B.; Barthlott, W. Diversity of Structure, Morphology and Wetting of Plant Surfaces. Soft Matter 2008, 4, 1943-1963.

(10) Hensel, R.; Neinhuis, C.; Werner, C. The Springtail Cuticle as a Blueprint for Omniphobic Surfaces. Chem. Soc. Rev. 2016, 45, 323-341.

(11) Tuteja, A.; Choi, W.; Ma, M.; Mabry, J. M.; Mazzella, S. A.; Rutledge, G. C.; McKinley, G. H.; Cohen, R. E. Designing Superoleophobic Surfaces. Science 2007, 318, 1618-1622.

(12) Tuteja, A.; Choi, W.; Mabry, J. M.; Mckinley, G. H.; Cohen, R. E. Robust Omniphobic Surfaces. Proc. Natl. Acad. Sci. USA 2008, 105, 18200-18205.

(13) Hensel, R.; Helbig, R.; Aland, S.; Braun, H.; Voigt, A.; Neinhuis, C.; Werner, C. Wetting Resistance at Its Topographical Limit: The Benefit of Mushroom and Serif T Structures. Langmuir 2013, 29, 1100-1112.

(14) Liu, T.; Kim, C. Turning a Surface Superrepellent even to Completely Wetting Liquids. Science 2014, 346, 1096-1100.

(15) Hensel, R.; Finn, A.; Helbig, R.; Braun, H.; Neinhuis, C.; Fischer, W.; Werner, C. Biologically Inspired Omniphobic Surfaces by Reverse Imprint Lithography. Adv. Mater. 2014, 26, 2029-2033.

(16) Kim, J. H.; Shim, T. S.; Kim, S. Lithographic Design of Overhanging Microdisk Arrays Toward Omniphobic Surfaces. Adv. Mater. 2016, 28, 291-298.

(17) Li, J.; Qin, Q. H.; Shah, A.; Ras, R. H. A.; Tian, X.; Jokinen, V. Oil Droplet Self-Transportation on Oleophobic Surfaces. Sci. Adv. 2016, 2, e1600148.

(18) Yun, G.; Jung, W.; Oh, M. S.; Jang, G. M.; Baek, J.; Kim, N.; Im, S. G.; Jung, H. SpringtailInspired Superomniphobic Surface with Extreme Pressure Resistance. Sci. Adv. 2018, 4, eaat4978. (19) Liu, X.; Gu, H.; Wang, M.; Du, X.; Gao, B.; Elbaz, A.; Sun, L.; Liao, J.; Xiao, P.; Gu, Z. 3D Printing of Bioinspired Liquid Superrepellent Structures. Adv. Mater. 2018, 30, 1800103.

(20) Li, Y.; Li, L.; Sun, J. Bioinspired Self-Healing Superhydrophobic Coatings. Angew. Chem. 2010, 122, 6129-6133.

(21) Jin, H.; Tian, X.; Ikkala, O.; Ras, R. H. A. Preservation of Superhydrophobic and Superoleophobic Properties upon Wear Damage. ACS Appl. Mater. Inter. 2013, 5, 485-488.

(22) Corral, I. B.; Calvet, J. V.; Salcedo, M. C. Use of Roughness Probability Parameters to Quantify the Material Removed in Plateau-Honing. Int. J. Mach. Tools Manufact. 2010, 50, 621-629.

(23) Hu, S.; Brunetiere, N.; Huang, W.; Liu, X.; Wang, Y. The Bi-Gaussian Theory to Understand Sliding Wear and Friction. Tribol. Int. 2017, 114, 186-191.

(24) Hu, S.; Reddyhoff, T.; Puhan, D.; Vladescu, S.-C.; Huang, W.; Shi, X.; Dini, D.; Peng, Z. BiGaussian Stratified Wetting Model on Rough Surfaces. Langmuir 2019, 35, 5967-5974.

(25) Hu, S.; Cao, X.; Reddyhoff, T.; Puhan, D.; Huang, W.; Shi, X.; Peng, Z.; Dini, D. ThreeDimensional Printed Surfaces Inspired by Bi-Gaussian Stratified Plateaus. ACS Appl. Mater. Inter. 2019, 11, 20528-20534. 
(26) Huovinen, E.; Takkunen, L.; Korpela, T.; Suvanto, M.; Pakkanen, T. T.; Pakkanen, T. A. Mechanically Robust Superhydrophobic Polymer Surfaces Based on Protective Micropillars. Langmuir 2014, 30, 1435-1443.

(27) Xiao, T. P.; Cifci, O. S.; Bhargava, S.; Chen, H.; Gissibl, T.; Zhou, W.; Giessen, H.; Toussaint, K. C.; Yablonovitch, E.; Braun, P. V. Diffractive Spectral-Splitting Optical Element Designed by Adjoint-Based Electromagnetic Optimization and Fabricated by Femtosecond 3D Direct Laser Writing. ACS Photonics 2016, 3, 886-894.

(28) Ergin, T.; Stenger, N.; Brenner, P.; Pendry, J. B.; Wegener, M. Three-Dimensional Invisibility Cloak at Optical Wavelengths. Science 2010, 328, 337-339.

(29) Medina-Sanchez, M.; Schwarz, L.; Meyer, A. K.; Hebenstreit, F.; Schmidt, O. G. Cellular cargo delivery: Toward assisted fertilization by sperm-carrying micromotors. Nano Lett. 2016, 16, 555-561.

(30) Klein, F.; Richter, B.; Striebel, T.; Franz, C. M.; von Freymann, G.; Wegener, M.; Bastmeyer, M. Two-Component Polymer Scaffolds for Controlled Three-Dimensional Cell Culture. Adv. Mater. 2011, 23, 1341-1345.

(31) Frenzel, T.; Kadic, M.; Wegener, M. Three-Dimensional Mechanical Metamaterials with a Twist. Science 2017, 358, 1072-1074.

(32) Hengsbach, S.; Lantada, A. D. Direct Laser Writing of Fractal Surfaces: Strategy to Design and Manufacture Textured Materials. Adv. Eng. Mater. 2015, 17, 172-180.

(33) Davis, E.; Liu, Y.; Jiang, L.; Lu, Y.; Ndal, S. Wetting Characteristics of 3-Dimensional Nanostructured Fractal Surfaces. Appl. Surf. Sci. 2017, 392, 929-935.

(34) Lantada, A. D.; Hengsbach, S.; Bade, K. Lotus-On-Chip: Computer-Aided Design and 3D Direct Laser Writing of Bioinspired Surfaces for Controlling the Wettability of Materials and Devices. Bioinspir. Biomim. 2017, 12, 066004.

(35) Quere, D. Fakir Droplets. Nat. Mater. 2002, 1, 14-15.

(36) Quere, D. Leidenfrost Becomes a Fakir. Nat. Mater. 2012, 11, 915-916.

(37) Marin, A. G.; Gelderblom, H.; Susarrey-Arce, A.; van Houselt, A.; Lefferts, L.; Gardeniers, J.

G. E.; Lohse, D.; Snoeijer, J. H. Building Microscopic Soccer Balls with Evaporating Colloidal Fakir Drops. Proc. Natl. Acad. Sci. USA 2012, 109, 16455-16458.

(38) Verho, T.; Korhonen, J. T.; Sainiemi, L.; Jokinen, V.; Bower, C.; Franze, K.; Franssila, S.; Andrew, P.; Ikkala, O.; Ras, R. H. A. Reversible Switching between Superhydrophobic States on a Hierarchically Structured Surface. Proc. Natl. Acad. Sci. USA 2012, 109, 10210-10213.

(39) Tsai, P.; Hendrix, M. H. W.; Dijkstra, R. R. M.; Shui, L.; Lohse, D. Microscopic Structure Influencing Macroscopic Splash at High Weber Number. Soft Matter 2011, 7, 11325-11333.

(40) Kim, H.; Lee, C.; Kim, M. H.; Kim, J. Drop Impact Characteristics and Structure Effects of Hydrophobic Surfaces with Micro- and/or Nanoscaled Structures. Langmuir 2012, 28, $11250-11257$.

(41) Laan, N.; de Bruin, K. G.; Bartolo, D.; Josserand, C.; Bonn, D. Maximum Diameter of Impacting Liquid Droplets. Phys. Rev. Appl. 2014, 2, 044018.

(42) Kim, H.; Park, U.; Lee, C.; Kim, H.; Kim, M. H.; Kim, J. Drop Splashing on a Rough Surface: How Surface Morphology Affects Splashing Threshold. Appl. Phys. Lett. 2014, 104, 161608. 
- TOC

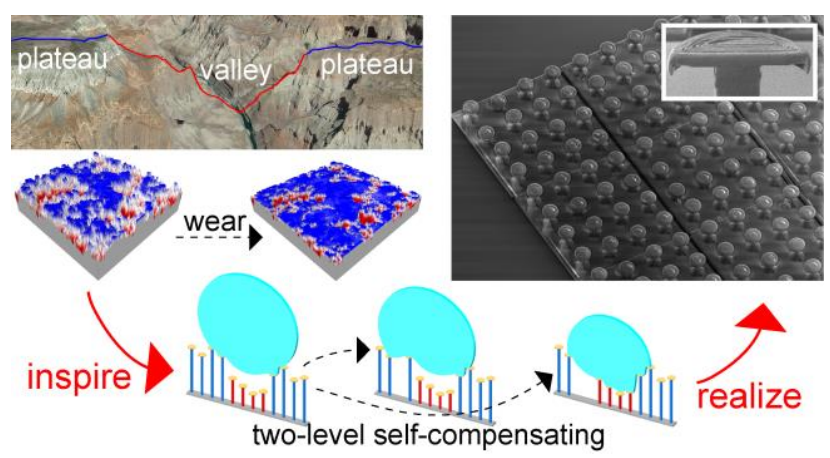

